\section{Pseudo-seizure in Seizure Disorder: A Case Report}

\author{
Dhana Ratna Shakya, MBBS, MD \\ Department of Psychiatry \\ BPKIHS, Dharan, Nepal
}

\author{
Address for correspondence: \\ Dhana Ratna Shakya, MBBS, MD \\ Department of Psychiatry \\ BPKIHS, Dharan, Nepal \\ Email: drdhanashakya@yahoo.com
}

Received, 22 November, 2011

Accepted, 24 December, 2011

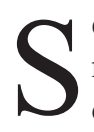
eizures are motoric, sensory, behavioral or psychic manifestation of hyper-synchronous electrical brain discharges. ${ }^{5}$ Some psychiatric disorders might show motoric, sensory, behavioral or other psychic symptoms simulating 'true seizure'., 3,57 Besides dissociative disorders; anxiety, depression, psychosis, for example, might have such symptoms. ${ }^{5,7,10}$

It is crucial to disentangle 'false' from 'true' seizure for effective management. ${ }^{2,3}$ It is, however frequently difficult and more confusing when both conditions cooccur. ${ }^{2,3}$ This issue has been more controversial with the
Pseudo-seizure may occur in seizure cases and it makes clinical decision difficult. Here is a case of 'true seizure' which later had 'pseudoseizure' in pretext of psychosocial stressors. This case-report intends to highlight this co-occurrence and need for managing both conditions.

A 22 year-unmarried female presented to psychiatry out-patient with spells of unresponsiveness and abnormal movements lasting for 1-3 minutes, suggestive of 'true seizure' both clinically and by electroencephalogram. She required $1600 \mathrm{mg}$ Sodium-valproate. After 6 month seizure control, she got married and then she had to stop medication. While stopping medicine, she had similar and the spells suggestive of complex partial seizure. The spells disappeared with $1200 \mathrm{mg}$ Oxcarbamazepine. After her divorce, she was frequently found to stare, and then either repeat incoherent words or remain unresponsive for 5-30 minutes, in others' presence and at times reported to faintly remember to try moving her hands. Those spells vanished while away from and recurred returning home because of her father's illness. Because of continued spells, she was forced to stop ongoing medication and seek traditional measures. Upon careful assessment, impression of 'dissociation in seizure' (seizure controlled then) was made. The antiepileptic was continued and psycho-social intervention made. Her later symptoms also gradually improved.

Key words: anti-epileptic, dissociation, pseudo-seizure, psychosocial intervention, seizure, stressor

report of some long term follow-up studies of 'pseudoseizure' many of which later were found to be associated with organic condition. ${ }^{6}$ This case displayed both 'true' and 'false seizures' which were managed with both antiepileptic and 'psycho-social interventions'.

\section{Case Report}

A 22 year unmarried, Buddhist, female certificate level student of a village in eastern Nepal presented to psychiatry out-patient with complaint of unresponsive spells. They 
were characterized by unconsciousness; fall down, abnormal jerky movements, frothing from inside mouth, urinary incontinence; occurrence also while alone and the history of injury and tongue bite during some spells. The spells were unpredictable; occurring anytime and anywhere: in market, garden, kitchen and road. She would gradually gain consciousness.

After 7 months of frequent attacks, she was brought to psychiatry OPD of a teaching hospital. CT-scan head revealed normal finding whereas electroencephalography (EEG) 'generalized seizure activity'. She responded to $1600 \mathrm{mg}$ Sodium-valproate, the dose reached with gradual optimization.

After 6 months of seizure control, she was married to a nearby village. In-laws were not happy about her taking medicine. After frequent questioning and confrontation, she stopped medication and follow-ups. She then started having similar 'generalized tonic clonic seizure' as before. In addition, she would stare for a while, then walk when she stumbled on the way, not leaving anything lying in her hand, either not responding to others' question or muttering some incomprehensible sounds and then falling into the ground subsequently with history of injury. It also occurred while in sleep and while alone. Because of uncontrolled symptoms; husband, in-laws and family members had to bring her again to the OPD. Considering changed clinical picture and the financial constraint, carbamazepine was started but because of intolerable sedation, oxcarbazepine was opted. In-laws and husband were not supportive; ultimately they divorced her. With 1200mg Oxcarbazepine, 'true seizure' disappeared but new though apparently similar symptoms emerged. She would stare, not speak but might walk for 5-10 minutes. During such spells, she never sustained any injury nor had incontinence. It occurred in presence of other people and did not occur when she went to her maternal uncle's home in Kathmandu. The spells appeared and were more frequent when she returned home because of her father's illness, possibly because of his heavy drinking. Because of the continued unresponsive spells, many villagers advised to stop medication and seek other traditional measures (which they had tried every sort before and at times apparently worked for some time). In dilemma, she was brought to the OPD service then by her friend.

Available history did reveal no significant head injury, CNS infection, similar or other major medical or psychiatric illness; neither in her past nor in her close blood relatives. There was no substance use. With clinical review, later features were deemed as 'pseudo-seizure'. Though she was worried about her father's condition and was not open about the divorce, there was no syndromal depression.

With the impression of 'dissociative conversion in case of seizure disorder' (seizure under control then), the same dose of Oxcarbazepine was continued and Clobazam was given for a short time. Her father was called for managing his drinking problem. Family intervention and psycho-education sessions were made with family, friend and patient. With gradual building of rapport, she was discussing and insightful about her stressor and disorder. She started following a scheduled activity to remain engaged; training on tailoring was useful for her. There was a gradual improvement in her symptoms.

\section{Discussion}

Seizure disorder or epilepsy is a common neuropsychiatric disorder with prevalence of about $1 \%{ }^{5}$ and with the 6 th position among the most important neuro-psychiatric causes of chronic disability. The features, phenomena, symptoms or psychopathology seen in 'true' seizures are varied widely; sensory, motor, altered consciousness, psychic to name some major ones. ${ }^{3,5}$ However, some features are relatively more consistently reported with 'true' seizures. Seizure like but 'false' seizures feature many psychiatric disorders and are referred to as 'pseudo-seizure' or 'psychogenic non-epileptic seizures'. Pseudo-seizures not only characterize dissociative disorders (ICD-10) or somatoform/dissociation conversion disorders (DSM-IV) but also co-occur with other psychiatric disorders, e.g. depression, anxiety and post-traumatic stress disorder. Pseudo-seizure or psychogenic non-epileptic seizures and dissociation conversion are among common presentations in clinical settings both as symptom and as diagnosis. ${ }^{4}$ 'False seizures' also might manifest with motor, sensory, altered consciousness or psychic (e.g. affective) symptoms which are at times really difficult to differentiate from seizure symptoms even for an experienced clinician. ${ }^{1,5}$

It is useful to carefully sort out certain reliable features for differentiating these two conditions (Table 1). ${ }^{5}$ Detailed history from the witness of spells is the mainstay as it has been for the presented case. Though not always, EEG is helpful for confirmation or for making differential diagnoses. CT/MRI scan-head may help rule-out specific causes. 'Prolactin estimation', available in our setting may help though not specific. ' Though 'video-EEG' is gold standard diagnostic method, it is not practically possible because of unavailability as in our case.

'True' seizure must be treated with anti-epileptic medication whereas the management of 'false' seizure is multimodal, including mainly psychosocial and sometimes medication. ${ }^{1}$ Treating 'false' seizure with antiepileptic is not only ineffective but also puts patient in unnecessary drug-burden and risk of adverse drug events. Similarly, treating 'true' seizure as 'false' will prove ineffective and may invite preventable complications of seizure. Therefore, it is important to sort out 'false' from 'true' seizure.

The decision making becomes more difficult when both the conditions occur in same person as in this case. Seizure and epilepsy are associated with a wide range of psychiatric disorders and they are reported more (20-60\%) than in general population. ${ }^{5}$ A Nepalese study among seizure cases referred to a psychiatric service showed $45 \%$ 
Pseudo-seizure in Seizure

\begin{tabular}{|c|c|}
\hline Nonepileptic Seizures/ Pseudo-seizure & Epileptic Seizures/ True-seizure \\
\hline \multicolumn{2}{|l|}{ Preceding ictus } \\
\hline Absence of explanatory disease or signs & Frequent evidence of neurological disease \\
\hline Anxiety auras: palpitations, choking, etc. & Wide range of epileptic auras \\
\hline Seizures may be induced or provoked & Rarely induced except for reactive seizures \\
\hline \multicolumn{2}{|l|}{ During ictus } \\
\hline Inconsistencies in clinical presentation & Fit specific seizure types \\
\hline Seizures may differ from attack to attack & Stereotypical seizure pattern \\
\hline Only occur when others are present & Often occur without witnesses or at night \\
\hline Gradual onset, prolonged duration ( $>2 \mathrm{~min}$ ) & Abrupt onset, short duration (<2 min) \\
\hline Asymmetrical movements, pelvic thrusts, and hyperarching & Decrescendo, symmetrical clonic activity in GTC seizure \\
\hline Rare whole body rigidity & Tonic rigidity at onset of GTC seizure \\
\hline Rare incontinence, tongue biting, self-injury & Incontinence, tongue biting if generalized \\
\hline Normal autonomic reactivity, corneal reflex, pupillary response & Disturbed autonomic reactivity, corneal reflex, pupillary response \\
\hline Avoids noxious stimuli or eye opening & Cannot avoid noxious stimuli \\
\hline Vocalizations may occur throughout ictus & Single vocalization, if present, at onset \\
\hline Normal ictal EEG & Abnormal ictal EEG \\
\hline \multicolumn{2}{|l|}{ After ictus } \\
\hline No postictal delirium & Typical postictal delirium \\
\hline No increase in prolactin & Prolactin $>1,000 \mathrm{IU} / \mathrm{L}, 10$ to 20 min postictally \\
\hline Normal postictal EEG & Postictal slowing on EEG \\
\hline Subsequent recall of events during ictus & No or fragmentary recall of ictal events \\
\hline No relationship of ictal frequency to anticonvulsant medications & Diminished seizure frequency with anticonvulsant medications \\
\hline EEG, electroencephalogram; GTC, generalized tonic-clonic. & \\
\hline
\end{tabular}

Table 1: Nonepileptic Seizures versus Epileptic Seizures ${ }^{5}$

with ICD-10 'mental and behavioral disorder' and the rest at least with some clinically significant symptom in 'Brief Psychiatric Rating Scale'. ${ }^{10}$ Depression, mania, ${ }^{9}$ anxiety and other neurotic (including dissociation conversion), psychotic disorders, personality and cognitive changes, suicide and self injurious behaviors ${ }^{8}$ were seen in these cases as reported elsewhere. ${ }^{11}$ This increases the chance of pseudo-seizure in seizure disorders. The co-occurrence of 'true' and 'false' seizures is reported variably, but remarkably high (10-50\%, even higher). ${ }^{1,2,3,6}$

Epilepsy in itself is a stressor, associated with stigma, psycho-social burden, possible adverse drug events and deficit of etiological CNS pathology, etc leading to higher bio-psycho-social etiological factors of psychiatric illness including dissociative disorders. ${ }^{4}$ The psycho-social stressor should be identified and duly addressed for management of co-morbid psychiatric condition besides seizure control, as in this case.

For seizure related psychopathology, seizure should be treated. Type of seizure, adverse effects and drug interactions mainly determine the choice of anti-epileptic drug (AED). Mono-therapy with adequate dose and duration is treatment principle as far as possible. AEDs with useful psychotropic effects, e.g. Sodium-valproate and 
Carbamazepine may be considered. While choosing a psychotropic for co-morbid psychiatric disorder or symptom, seizure friendly medication with less druginteraction propensity is considered. In this case, clobazam was added to Oxcarbazepine for the control of additional symptom. 'Psychogenic non-epileptic', 'false' or 'pseudoseizure' deserves attention: proper diagnosis, work up of possible factors, drug and non-drug therapy accordingly when present in seizure cases besides seizure control with anti-epileptic medicine.

\section{References}

1. Auxéméry Y, Hubsch C, Fidelle G. Psychogenic non epileptic seizures: a review. Encephale 37:153-158, 2011

2. Carreno M. Recognition of Nonepileptic Events. Seminars in Neurology 28:297-304, 2008

3. Kaufman DM. Epilepsy. In: Clinical Neurology for Psychiatrists. Philadelphia: Sounders Elsevier, 2007, pp 203-240.

4. Lesser R. Treatment and Outcome of Psychogenic nonepileptic seizures. Epilepsy currents 3: 198-200, 2003

5. Mendez MF. Neuropsychiatric Aspects of Epilepsy. In: Sadock BJ, Sadock VA and Ruiz P. Comprehensive
Textbook of Psychiatry. $9^{\text {th }}$ edition. Philadelphia: Wolters Kluwer/ Lippincott Williams \& Wilkins; 2009. pp 451-62.

6. Reuber M, Pukrop R, Bauer J, Helmstaedter C, Tessendorf N, Elger CE: Outcome in psychogenic nonepileptic seizures: 1 to 10 -year follow-up in 164 patients. Ann Neurol 53: 305, 2003

7. Ristic AJ, Petrovi? I, Vojvodic N, Jankovic S, Sokic D. Phenomenology and psychiatric origins of psychogenic non-epileptic seizures. Srp Arh Celok Lek 132: 22-27, 2004

8. Shakya DR, Shyangwa PM, Pandey AK, Subedi S, Yadav S. Self Injurious Behavior in Temporal Lobe Epilepsy. J Nepal Med Assoc 49: 239-242, 2010

9. Shakya DR, Shyangwa PM, Shakya R, Maskey R, Lamichhane N. Bipolar mood in seizure disorders: A Case report. Nepal Journal of Neuroscience 1: 1517,2010

10. Shakya DR. Psychiatric associations/ manifestations in seizure disorders. Asian J Psychiatry 4: S62, 2011

11. Swinkels WAM, Kuyk J, van Dyck R, Spinhoven Ph. Psychiatric comorbidity in epilepsy. Epilepsy Behav 7: 37,2005 